Z Herz-Thorax- Gefäßchir 2008 · 22:91-93 DOI 10.1007/s00398-008-0620-x Online publiziert: 4. April 2008

(c) Springer Medizin Verlag 2008

\author{
K. H. Leitz ·. Neu \\ Schlichtungsstelle für Arzthaftpflichtfragen, Hannover, BRD
}

\title{
Druckschaden der Haut nach Herzoperation
}

chirurgiegerät trug die Inventarnummer ID 770. Bei den Wartungen im Dezember 2004 sowie im November 2005 waren keinerlei Beanstandungen festgestellt worden.

Im Nachtdienst vom 9. auf den 10. Februar 2005 fiel eine $4 \times 2 \mathrm{~cm}$ große Rötung im Bereich des linken Gesäßes auf. Diese wurde mit Mirfulan behandelt. Am 10. Februar 2005 wurde der Defekt als bläulich livide Verfärbung der Größe $6,5 \times 2,5 \mathrm{~cm}$ beschrieben. Am nächsten Tag erfolgte die Eröffnung einer Blase. Im Verlauf desselben Tages wurde ein oberflächlich geröteter, nicht belegter Hautdefekt dokumentiert und mit Allevyn behandelt. Am 12. Februar 2005 verzeichnet die Dokumentation einen $6,5 \times 2,5 \mathrm{~cm}$ großen Dekubitus II. Der letzte Eintrag beschreibt einen $5 \times 5 \mathrm{~cm}$ großen, trockenen, nicht belegten Hautdefekt.

Am 16. Februar 2005 erfolgte die Verlegung in eine Medizinische Klinik, von wo der Patient am 21. Februar 2005 nach Hause entlassen wurde.

Am 25. Februar 2005 erfolgte wegen Wundheilungsstörungen im Sternalbereich sowie Sternuminstabilität die erneute Aufnahme im Herzzentrum. Am 27. Februar 2005 wurde eine Sternumrevision mit Refixation und Einlage einer Spül-Saug-Drainage vorgenommen. Der Operationsbericht spricht von einer floriden Mediastinitis. Die Revision dauerte 65 Minuten. Der Patient lag erneut auf dem Rücken. Der intraoperative Abstrich ergab Staphylococcus aureus.
Gespült wurde die Sternumwunde über 3 Tage, danach wurde 3 Tage lediglich gesaugt und an 3 aufeinander folgenden Tagen ein Abstrich genommen. Die zwei Redondrainagen sowie drei Thoraxdrainagen verblieben insgesamt 9 Tage.

Bei Aufnahme auf der Intensivstation am 27. Februar 2005 war der Dekubitus als im Stadium IV befindlich beschrieben. Die Oberfläche sei schwarz gewesen. Vom 28. Februar bis 4. März 2005 wurde der Patient auf einer AtmosAir-Matratze der Firma KCI gelagert. Von da an kam eine ProfiCARE-Matratze derselben Firma zum Einsatz. Am 19. März 2005 wurde eine schwarze Nekrose abgetragen. In der Dokumentation vom 21. März 2005 zeigten sich keine Beläge mehr, die Größe der Wunde wurde mit $3 \times 4,5 \mathrm{~cm}$ angegeben. Am 23. März 2005 konnte der Patient entlassen werden, nachdem noch eine Bronchoskopie eines verdächtigen computertomographischen Befundes wegen durchgeführt worden war. Laut Schreiben des Patientenvertreters kam es erst im Juli 2005 zum vollständigen Abheilen der Wunde.

Unter den Unterlagen war ein Gutachten des Medizinischen Dienstes, in dem es heißt: „Die Gewebsschäden am linken Gesäß sind mit an Sicherheit grenzender Wahrscheinlichkeit auf eine fehlerhafte Stromableitung bei Einsatz des für diese Operation erforderlichen Elektrochirurgiegerätes zurückzuführen." Das Gutachten wurde von einem Facharzt für Chirurgie und Unfallchirurgie erstellt. 
Der den Ärzten des Herzzentrums gemachte Vorwurf wird mit einer Sorgfaltspflichtverletzung begründet, die zu einem Hautschaden am linken Gesäß führte.

Diesem Vorwurf widersprechend verteidigen sich die Ärzte des Herzzentrums mit der Einhaltung aller Regeln der ärztlichen Sorgfaltspflicht. Bei diesem Patienten seien beide Beine mit Desinfektionslösung abgewaschen worden. Durch spezielle, nach dem Desinfektionsvorgang entfernte Saugunterlagen habe man jedoch Vorsorge getroffen, damit das Desinfektionsmittel nicht unter das Gesäß habe rinnen können.

Der von der Schlichtungsstelle beauftragte, externe herzchirurgische Gutachter kommt zu folgender Bewertung:

1. Die kombinierte Gefäß- und Koronaroperation wurde entsprechend geltendem Standard durchgeführt.

2. Bei Eingriffen mit der Herz-LungenMaschine besteht ein erhöhtes Risiko für einen intraoperativ gesetzten Hautschaden, trotz sorgfältigem Vorgehen.

3. Intraoperativ war die Lagerung korrekt.

4. Die postoperative Betreuung einschließlich der Behandlung des Hautdefektes war fachgerecht.

Zusammenfassend kommt der Gutachter zur Bewertung, dass eine Verletzung der ärztlichen Sorgfaltspflicht weder eindeutig auszuschließen, noch festzustellen sei.

In der Stellungnahme des Patientenvertreters wird das Fehlen einer Begründung für die Annahme, dass trotz sorgfältigen ärztlichen Vorgehens ein erhöhtes Risiko bestünde, kritisiert.

Der Bewertung durch die Schlichtungsstelle seien zunächst allgemeine Ausführungen vorangestellt.

Von einem Hautschaden lässt sich stets nur sehr schwer auf dessen Ursache schließen, der Hautschaden selbst sagt nichts über die Art der Energie, die auf die Haut wirkte, sei es mechanische Energie als Druck-, Reibungs- oder Scherkräfte, thermische Energie in Form von Hitze oder Kälte bzw. chemische oder Strahlungsenergie. Denkbar sind auch Summationseffekte verschiedener Energieformen bei- spielsweise in Form von thermischer und chemischer Energie, deren Zusammenwirken eine Klärung der Ursache noch schwieriger macht.

Deshalb kommt es zu der in den Unterlagen anzutreffenden Begriffsvielfalt. Einerseits wird von einem Verbrennungsschaden gesprochen und dabei an Verbrennungen durch das benutzte Elektrochirurgiegerät gedacht, so der Gutachter des Medizinischen Dienstes. Andererseits gehen die in Anspruch genommenen Ärzte des Herzzentrums von einem Druckschaden aus. Auch der Patientenvertreter führt aus, dass es im Wesentlichen um die Frage nach der Ursache des Hautschadens bei diesem Patienten gehe.

Die durch welche Energieform auch immer geschädigte Haut reagiert stets gleich. Der Rötung folgen Blasenbildung, danach Zerstörung der gesamten Haut sowie des subkutanen Gewebes. Die Tiefe des Hautschadens ist dabei abhängig von der Energiehöhe pro Zeit.

Natürlich existieren Krankheiten, welche die Entstehung eines Hautschadens begünstigen. Patienten mit neurologischen Störungen, aber auch Kranke mit arterieller Verschlusskrankheit gehören zu den Risikopatienten hinsichtlich eines intraoperativen Hautschadens.

In diese Risikogruppe gehören auch Patienten mit langen chirurgischen Eingriffen, wie beispielsweise Herzoperationen. Gerade diese Patienten sind längerer Druckbelastung, Kälteeinwirkung und einer Durchblutungsstörung angesichts des maschinenbedingten verringerten Herzminutenvolumens ausgesetzt. Darüber hinaus bringen sie infolge ihrer generalisierten Arteriosklerose inklusive Verengungen in vorgeschalteten Gefäßen Faktoren mit, die die Leistungsbreite der Hautgefäße zusätzlich kompromittieren können. Schließlich müssen während Herzoperationen Medikamente appliziert werden, die ebenfalls eine konstringierende Wirkung auf die Hautgefäße aufweisen.

Der Literatur und beiden Gutachten nach gilt zwar, dass bei einem perioperativ gesetzten Hautschaden dem ersten Anschein nach von einer Sorgfaltspflichtverletzung auszugehen sei, dass dieser Schluss jedoch nicht zulässig ist, wenn besondere Umstände und Tatsachen vorliegen, die ihrerseits ebenso gut für die Entstehung der Hautläsion verantwortlich sein könnten. Herzoperationen fallen aus den oben genannten Gründen per se unter die hier gemeinten besonderen Umstände und Tatsachen. Der Gutachter des MDK konnte als Facharzt für Chirurgie und Unfallchirurgie die Besonderheiten einer herzchirurgischen Operation nicht hinreichend bewerten.

Demnach kommt die Schlichtungsstelle zu folgender Bewertung:

Der Einsatz des Elektrochirurgiegeräts war den Unterlagen nach fachgerecht, die Wartungsmaßnahmen entsprechend des Medizinproduktegesetzes wurden eingehalten und das Gerät nicht beanstandet. Hinweise für eine fehlerhafte Platzierung der Elektroden (zu großer Abstand, falsche Wahl des Ortes) fehlen.

Die Schlichtungsstelle geht von einem Druckschaden aus, der durch die erste, 266 Minuten dauernde Operation, eingeleitet, durch die zweite Operation mit lang anhaltender Immobilisation aufgrund des zur Infektbekämpfung notwendigen SpülSaug-Vorgangs aber zusätzlich verstärkt wurde und deshalb erst im Juli 2005 zur Abheilung kam.

Die Schlichtungsstelle sieht, da die Grundsätze des Anscheinsbeweises hier nicht zur Anwendung gelangen können, keinen Beweis eines Behandlungsfehlers und folgt dem Gutachter in seiner Meinung, dass bei kardiochirurgischen Operationen mit der Herz-Lungen-Maschine trotz sorgfältigsten ärztlichen Vorgehens ein erhöhtes Risiko für einen intraoperativen Hautschaden besteht.

Bei dem hier aufgetretenen Hautschaden handelt es sich um eine Komplikation, die auch auftreten könnte, wenn, wie hier, ärztlicherseits nach den Standards des Fachs vorgegangen wurde. Ohne entsprechende Hinweise kann das Auftreten der Komplikation allein deshalb hier nicht als Beweis für fehlerhaftes ärztliches Vorgehen gewertet werden.

Die Schlichtungsstelle hielt Schadensersatzansprüche nicht für begründet. 


\section{Fazit}

1. Die häufigste Ursache einer intraoperativ gesetzten Hautläsion sind mechanische Kräfte (Druckschaden). Deshalb sollte primär stets von dieser Diagnose ausgegangen werden.

2. Eine exakte Dokumentation der intraoperativen Lagerung, der Anbringung der Neutralelektrode sowie des Wund-

\section{Korrespondierender Autor}

\section{Prof. Dr. med. K. H. Leitz}

Barlachweg 23, 28355 Bremen, BRD

E-Mail:kh.leitz@t-online.de

Interessenkonflikt. Der korrespondierende Autor gibt an, dass kein Interessenkonflikt besteht. verlaufs (eventuell mit Fotodokumentation) seitens des behandelnden Arztes ist die einzige Möglichkeit, sich gegen den Vorwurf einer Verletzung der gebotenen Sorgfaltspflicht zu wehren.

3. Die Wunddokumentation ist Aufgabe des Arztes und nicht des Pflegepersonals.
Z Herz-Thorax- Gefäßchir 2008 · 22:91-93 DOI 10.1007/s00398-008-0620-x

(c) Springer Medizin Verlag 2008

\section{K. H. Leitz · J. Neu}

\section{Druckschaden der Haut nach Herzoperation}

\section{Zusammenfassung}

Im nachfolgenden Artikel wird über einen Sakraldekubitus nach Herzoperation berichtet, der durch eine sekundäre Sternumdehiszenz verstärkt wurde und erst nach fünf Monaten zur Abheilung kam. Das Vorliegen eines Behandlungsfehlers wird verneint.

\section{Schlüsselwörter}

Dekubitus · Herzchirurgie · Behandlungsfehler

\section{Decubitus after open heart surgery}

\section{Abstract}

A case of a sacral decubitus after open heart surgery is presented, which increased by a secondary sternal dehiscence. A final healing was achieved only after five months. A malpractice claim is denied.

\section{Keywords}

decubitus · heart surgery · malpractice 